\title{
Vertically Resolved Convective/Stratiform Echo Type Identification and Convectivity Retrieval for Vertically Pointing Radars
}

\author{
Ulrike Romatschke, ${ }^{a}$ Michael J. Dixon, ${ }^{a}$

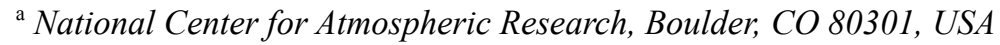 \\ Corresponding author: Ulrike Romatschke, romatsch@ucar.edu
}

This work has not yet been peer-reviewed and is provided by the contributing authors as a means to ensure timely dissemination of scholarly and technical work on a noncommercial basis. Copyright and all rights therein are maintained by the authors. It is understood that all persons copying this information will adhere to the terms and constraints invoked by each author's copyright. This work may not be reposted without explicit permission of the copyright owner. 


\title{
Vertically Resolved Convective/Stratiform Echo Type Identification and Convectivity Retrieval for Vertically Pointing Radars
}

\author{
Ulrike Romatschke, ${ }^{a}$ Michael J. Dixon, ${ }^{a}$

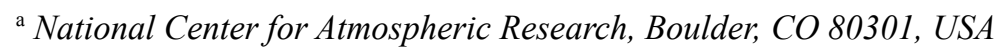 \\ Corresponding author: Ulrike Romatschke, romatsch@ucar.edu
}

\section{Abstract}

Using data from the airborne HIAPER Cloud Radar (HCR), a partitioning algorithm (ECCO-V) that provides vertically resolved convectivity and convective vs stratiform radar echo classification is developed for vertically pointing radars. The algorithm is based on the calculation of reflectivity and radial velocity texture fields that measure the horizontal homogeneity of cloud and precipitation features. The texture fields are translated into convectivity, a numerical measure of the convective or stratiform nature of each data point. The convective/stratiform classification is obtained by thresholding the convectivity field. Subcategories of low, mid, and high stratiform, shallow, mid, deep, and elevated convective, and mixed echoes are introduced, which are based on the melting layer and divergence level altitudes.

As the algorithm provides vertically resolved classifications, it is capable of identifying different types of vertically-layered echoes, and convective features that are embedded in stratiform cloud layers. Its robustness was tested on data from four HCR field campaigns which took place in different meteorological and climatological regimes. The algorithm was adapted for use in space borne and ground based radars, proving its versatility, as it is adaptable not only to different radar types and wavelengths, but also different research applications. 


\section{Introduction}

The terms convective and stratiform are used to describe different types of clouds and precipitation. Convective processes that lead to convective clouds and precipitation are characterized by vertical air motions which equal or exceed the fall speed of frozen hydrometeors when averaged over a certain horizontal area (Houze 2014). In radar echoes they are depicted as vertically oriented columns of high reflectivity. In regions with stratiform clouds and precipitation the vertical air motion is generally small compared to the fall speed of frozen hydrometeors. The particles are suspended mid-air and appear as horizontally uniform layers in radar images. At the melting layer, which is generally located up to a few hundred meters below the $0{ }^{\circ} \mathrm{C}$ freezing level (Romatschke 2021), the so-called radar bright band is often visible as a narrow horizontal layer of intense echo. A discontinuity in the radar radial velocity field at the melting layer indicates an increase in vertical velocity (Houze 2014 ) as the particles change from the frozen to the liquid phase.

Since convective and stratiform clouds and precipitation indicate fundamentally different physical and microphysical processes, and since radar is a primary tool for distinguishing these two categories of clouds and precipitation, there is a long history of automating the detection and separation of convective and stratiform radar echo types. Many of these algorithms rely on identifying the characteristics of the horizontal vs vertical structure of the echoes (Steiner et al. 1995; Biggerstaff and Listemaa 2000; Awaka et al. 1997; Powell et al. 2016). Others use machine learning techniques, (Anagnostou 2004; Yang et al. 2013; Wang et al. 2021). Although, on a fundamental level, convective and stratiform processes are defined based on vertical air motions, most partitioning algorithms rely on clues from reflectivity, since observations of vertical velocity are rarely available to the scientist.

Previously convective and stratiform echo classifications were only provided on the horizontal dimensions so that all points in a vertical radar echo column would be classified identically. The recently introduced ECCO (Echo Classification from COnvectivity) algorithm by Dixon and Romatschke (2022) provides echo type that is resolved in both the vertical and horizontal dimensions. The ECCO algorithm was developed for three dimensional radar grids and two dimensional grids on the horizontal axes. We use data from an airborne cloud radar to adapt the original ECCO algorithm (which we will call ECCO-3D hereafter) for vertically pointing radars (which we will call ECCO-V hereafter), that is, for two dimensional radar grids with a vertical and a horizontal (or time) axis. ECCO-V is then demonstrated using data from space borne and ground based radars. As vertically pointing radars can only sample vertical cross sections through storms, adding vertically resolved echo type classifications is of particular interest, because otherwise the convective/stratiform classification is limited to only one dimension on the horizontal (time) axis (Haynes 2018). An additional advantage of the ECCO method is that it not only provides a vertically resolved classification but it also introduces the concept of convectivity, a quantitative measure of how 
convective a certain grid location is, as opposed to the purely qualitative binary classification of either convective or stratiform for a vertical column provided by previous methods.

\section{Data}

The High-performance Instrumented Airborne Platform for Environmental Research (HIAPER) is a Gulfstream V aircraft deployed in field campaigns by the National Center for Atmospheric Research (NCAR) for the National Science Foundation (NSF). One of the instruments that can be requested by the scientific community on the aircraft is the HIAPER Cloud Radar (HCR), a 94-GHz (W-band) cloud radar which is mounted in an underwing pod (Vivekanandan et al. 2015; Romatschke et al. 2021). HCR has polarimetric and Doppler capabilities and, among other variables, provides the first two standard radar moments reflectivity (DBZ) and radial velocity (VEL), both of which are used in ECCO-V. Melting layer altitude detections (Romatschke 2021) and temperature reanalysis data (European Centre for Medium-Range Weather Forecasts) interpolated onto the HCR time-range grid (Romatschke et al. 2021) are also included in the standard HCR data sets. HCR samples at a range resolution of $\sim 20 \mathrm{~m}$ and a time resolution of $10 \mathrm{~Hz}$, which at typical aircraft speed translates to a $\sim 20 \mathrm{~m}$ horizontal resolution, i.e. effectively a 20 -by-20 $\mathrm{m}$ grid spacing.

HCR has been deployed in several major field campaigns ranging in location from the tropics to the Southern Ocean (Albrecht et al. 2019; McFarquhar et al. 2020; Fuchs-Stone et al. 2020), sampling a variety of cloud types from large stratocumulus fields to deep tropical convection. ECCO-V was developed with HCR datasets and tested on data from all major HCR field campaigns to ensure its adaptability to different cloud types and meteorological regimes.

To broaden the applicability of the algorithm, we adapted it for other types of radar. The space borne Cloud Profiling Radar (CPR, Im et al. 2005) onboard the CloudSat satellite is an ideal candidate for the deployment of this convective/stratiform echo type algorithm. Like $\mathrm{HCR}$, it is a 94-GHz nadir pointing W-band radar. It samples clouds around the globe with a vertical resolution of $500 \mathrm{~m}$ and an along-track resolution of $1.7 \mathrm{~km}$. We use radar reflectivity from the 2B-GEOPROF product (Marchand et al. 2008) from July 4, 2006.

A version of the algorithm suitable for ground based vertically pointing cloud radars was tested on the millimeter wavelength cloud radar (MMCR) system located at the Atmospheric Radiation Measurement (ARM) Southern Great Plains (SGP) facility in Oklahoma, US. MMCR is a zenith pointing 35-GHz Ka-band radar, and we use reflectivity data from scan mode 3, which was collected on August 17, 2008, at an $\sim 87 \mathrm{~m}$ vertical and a $\sim 5 \mathrm{~s}$ time resolution.

See Data Availability Statement for information on how to access the different datasets. 


\section{Algorithm description}

As outlined in the introduction, convective radar echoes show vertically oriented features while stratiform echoes present as horizontally uniform layers. Hence, a good approach to identifying convective regions is to test how homogeneous or heterogeneous the radar echoes are on the horizontal axis. Following the ECCO-3D method, we calculate a texture field on the horizontal time dimension as outlined in the following.

We start with DBZ and complete one preprocessing step where we fill in all clear air regions with reflectivity values from their closest non-missing neighbor in the time dimension, to avoid artifacts at the outer edges of the cloud echoes.

Reflectivity texture is calculated over a certain horizontal extent and the following operations are performed on specified time segments in the time dimension. For the HCR grid resolution, a running window over $10 \mathrm{~s}$ time segments gives good results. To make sure all contributions in the texture field are from true heterogeneities and not from slopes in the reflectivity field, we first remove the gradient in each $10 \mathrm{~s}$ segment by calculating a fit to the segment of the form:

$$
D B Z_{f i t}=a x+b
$$

where $a$ is the slope of the fit line, $b$ is the intercept, and $x$ represents the time (or space) dimension. We calculate the corrected reflectivity by subtracting the fit from the measured values and adding the mean:

$$
D B Z_{\text {corr }}=D B Z_{-D i t}+D B Z_{\text {mean }}
$$

where $D B Z$ is the measured reflectivity, and $D B Z_{\text {mean }}$ is the mean reflectivity over the time segment. Adding the mean reflectivity is important as it retains the information of the strength of the radar echo.

Before we proceed to calculate reflectivity texture, we adjust the $D B Z_{\text {corr }}$ values by subtracting a base reflectivity $\left(D B Z_{\text {base }}\right)$

$$
D B Z_{\text {adj }}=D B Z_{\text {corr }}-D B Z_{\text {base }} \text {. }
$$

This is necessary because HCR reflectivity values are often negative and the calculation of reflectivity texture involves taking the square root of quadratic values (Eq. 4 below) and therefore cannot handle negative values unambiguously. For HCR, $D B Z_{\text {base }}$ was chosen experimentally and set to $-10 \mathrm{dBZ}$. We set $D B Z_{\text {adj }}$ values that are smaller than $1 \mathrm{dBZ}$ to 1 before we calculate reflectivity texture $T D B Z$ as

$$
T D B Z=\sqrt{\operatorname{stdev}\left(D B Z_{a d j}^{2}\right)}
$$

where stdev is the standard deviation over the time segment. 
The ECCO-3D algorithm successfully uses $T D B Z$ alone but since the fundamental definition of convective vs stratiform processes is based on vertical air motion, and HCR radial velocity can be viewed as a proxy, we also calculate velocity texture (TVEL) and integrate it into the ECCO-V version of the algorithm. (Note that for radars that do not provide radial velocity, or when its use is inconvenient for other reasons, ECCO-V can be used with TDBZ alone.) TVEL is calculated in the same way as TDBZ. However, the base value that is subtracted from VEL is set to $-20 \mathrm{~m} \mathrm{~s}^{-1}$ to ensure that no negative velocity values are present. In an additional preprocessing step, each contiguous cloud echo is slightly eroded to avoid artifacts from noise at the cloud edges. Note that velocity folding needs to be taken into account when calculating TVEL.

The concept of convectivity was introduced in ECCO-3D as a numeric measure of the convective vs stratiform nature of each grid location. It ranges from 0 to 1 , where 0 represents purely stratiform and 1 represents purely convective echo. To map TDBZ and TVEL to a common convectivity field we first scale them by dividing them by $12 \mathrm{dBZ}$ for TDBZ and 5 $\mathrm{m} \mathrm{s}^{-1}$ for TVEL. Note that these scaling values were found experimentally and will vary for different radars. We then multiply the scaled TDBZ and TVEL fields with each other and set values greater than 1 to 1 . If radial velocity is not available, convectivity can be calculated from $T D B Z$ alone by omitting the multiplication step. However, we found that combining both fields leads to better accuracy and better detection of small scale convective features.

An example of DBZ, VEL, and convectivity sampled on September 27, 2019, from 12:30 to 12:51 UTC off the coast of Costa Rica during the Organization of Tropical East Pacific Convection (OTREC) field campaign (Fuchs-Stone et al. 2020) is shown in Fig. 1a-c. In this case HCR sampled a mid-to-high level stratiform cloud with new convective cells forming below. Convectivity is high in the developing convective cells and mostly low in the stratiform cloud but small-scale regions of high convectivity are embedded within the stratiform cloud (Fig. 1c).

A basic classification into convective, mixed, and stratiform echo is achieved by simply setting thresholds on the convectivity field. Following the ECCO-3D method, we set a threshold of 0.4 to separate stratiform from mixed echo and a threshold of 0.5 to separate mixed from convective echo. We perform a few post-processing steps, which are unique to ECCO-V, to improve the classification. First, we remove mixed and convective features that are smaller than a certain number of contiguous grid points (500 for HCR). The second post-processing step is specific to stratiform precipitation. While in cloud echo above the melting layer, stratiform echo in general presents as homogeneous horizontal layers, that is not necessarily the case for stratiform precipitation below the melting layer. The strength of precipitation can vary significantly over short horizontal distances which results in vertically-oriented reflectivity structures that could be mis-classified as convective echo by the algorithm. To identify these potential mis-classifications we check each mixed and convective feature and find those that fulfill both of the following criteria: (a) they are mostly located below the melting layer; and (b) they are located below a thick layer of stratiform 
echo aloft. Echo features that fulfill these criteria are set to stratiform. For the remaining mixed and convective contiguous features, we perform morphological dilation followed by morphological closing to join features that are in close proximity. During these operations we make sure not to dilate into stratiform regions that are not vertically connected. An example of the basic classification resulting from the thresholding and the post-processing steps is shown in Fig. 1d. The algorithm correctly identifies both the stratiform and convective clouds but also highlights elevated small-scale convective features within the stratiform cloud.
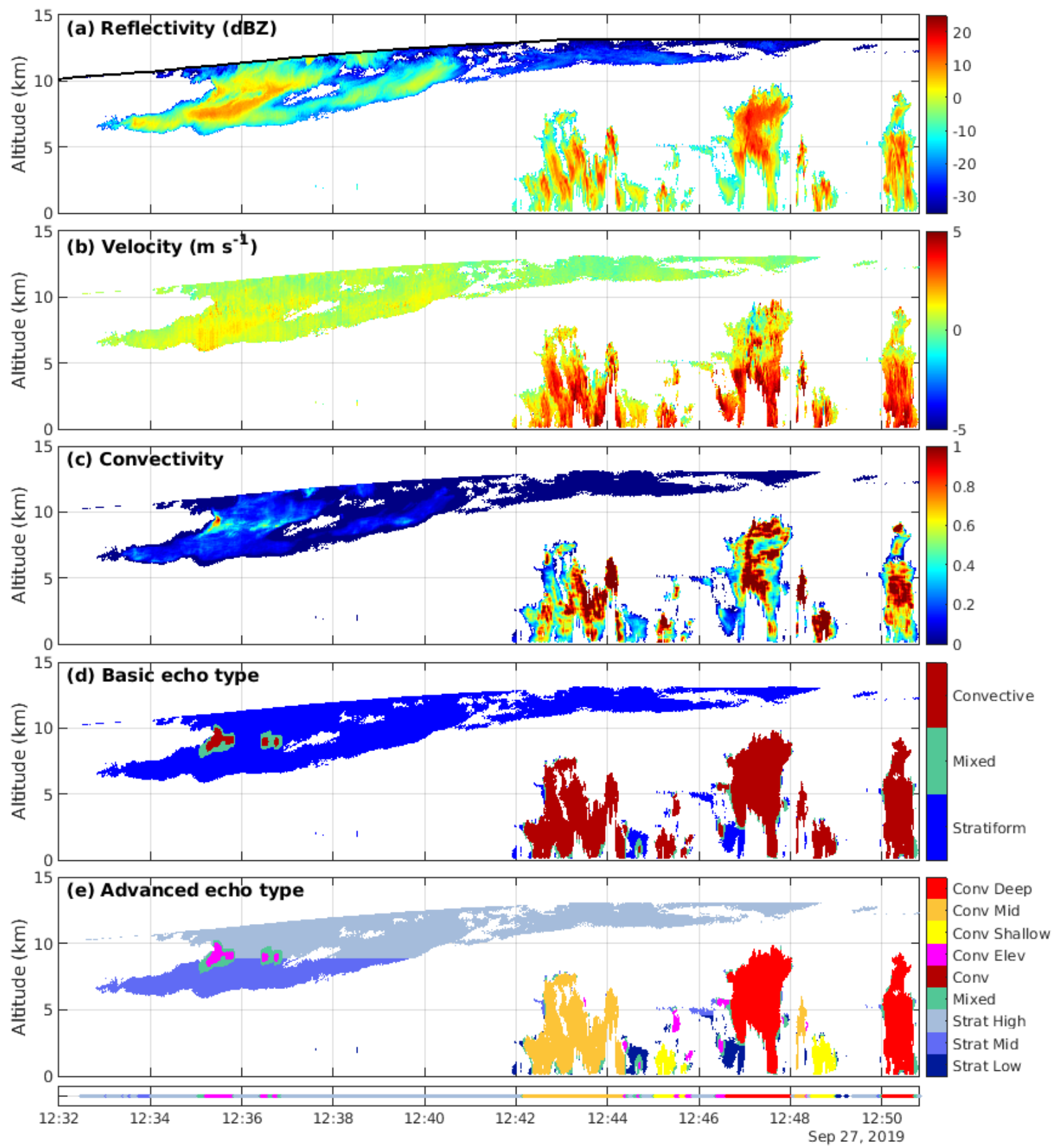

Fig. 1: (a) Reflectivity, (b) radial velocity, (c) convectivity, (d) basic and (e) advanced echo type from HCR data sampled on September 27, 2019 from 12:32 to 12:51 UTC during the OTREC field campaign. The altitude of the aircraft is shown as a black line in (a). 
For certain applications, convectivity or the basic echo type classification may provide all needed information. However, to provide additional information on the convective or stratiform echo, we perform a secondary advanced sub-classification, again following the ECCO-3D method. For this advanced classification, the atmosphere is divided into three regions: The low and warm region below the melting layer (which is provided in the HCR datasets, Romatschke et al. 2021), the mid-region above the melting layer but below the divergence level, and the high region above the divergence level. Following ECCO-3D, we set the divergence level to coincide with the $-25^{\circ} \mathrm{C}$ isotherm. Stratiform echo is divided into low, mid, and high stratiform echo accordingly. Convective features with echo tops below the melting layer are classified as shallow convective echoes. If they extend above the melting layer but top out below the divergence level they are classified as mid convective, and if they extend above the divergence level they are classified as deep convective echoes. Convective features that do not extend downward to close to the surface are classified as elevated convective echoes, with one exception: If the convective feature is located directly above an area where the radar signal is completely attenuated (marked by a quality flag of extinct, Romatschke et al. 2021), it is assumed to reach all the way to the surface and is classified accordingly. Especially in heavy convection, echo extinction at W-band is quite frequent, e.g. under the deep convective feature at 12:47 UTC in Fig. 1. When the aircraft penetrates a cloud and HCR only samples a part of the cloud as it points either nadir or zenith, it is sometimes not possible to decide to which of the convective categories a convective feature belongs. For example, the feature top may be unknown if HCR is pointing nadir. In such cases the feature retains its convective classification from the basic partitioning without the addition of an advanced category. This limitation does not apply to space borne or ground based applications as such radars generally sample whole clouds. The final classification with all its sub-categories is shown in Fig. 1e. The algorithm labels convective cells in their very early stages as shallow (12:45:30 and 12:48:40), growing convective cells as mid (12:42-12:44), and mature convective cells as deep (12:47-12:48 and 12:50 to 12:51). The stratiform cloud is divided into a mid and a high part and the small scale embedded convective features are labeled as elevated convective echoes.

As a final step we collapse the final classification into the traditional classification used by previous algorithms where each vertical column is assigned a single classification. A useful one-dimensional classification along the time axis is created by assigning classification values in the two dimensional classification in a thoughtful way. Since most users will likely be interested in whether convective echo exists anywhere in the vertical column, we give convective echo precedence over stratiform echo. We therefore assign the classification flag values in the two-dimensional classification in the following ascending order, which represents a prioritization from low to high: low, mid, high stratiform echo, mixed echo, elevated, shallow, mid, deep convective echo. We take the maximum value in each vertical column to derive the one-dimensional classification shown as a colored line in Fig. 1e. (This prioritization can easily be changed based on user needs.) 

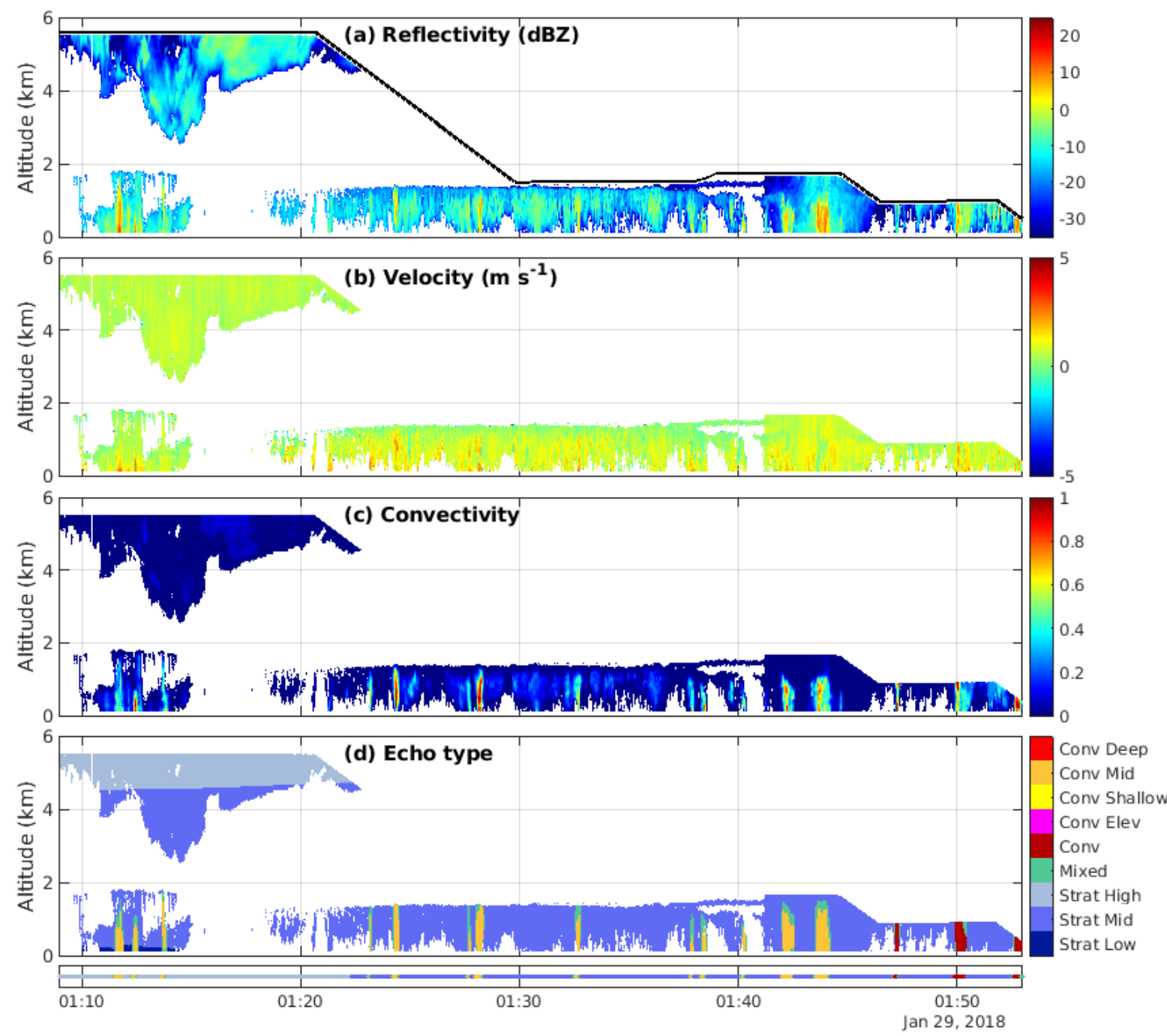

Fig. 2: (a) Reflectivity, (b) radial velocity, (c) convectivity, and (d) advanced echo type from HCR data sampled on January 29, 2018, from 01:09 to 01:53 UTC during the SOCRATES field campaign. The altitude of the aircraft is shown as a black line in (a).

\section{Results}

\subsection{HCR field campaigns}

As mentioned earlier (Section 3), the algorithm was developed on HCR observations and extensively tested and adjusted to provide realistic results, as determined by a human expert, for observations from all of the major HCR field campaigns to date. Since, to our knowledge, this is the first vertically resolving echo type classification algorithm for vertically pointing radars, there is no direct way to evaluate its performance. The original ECCO-3D method was thoroughly statistically evaluated and the results compared well with previous algorithms and lightning data. We expect ECCO-V to perform similarly. However, as with all echo type classification algorithms, some mis-classifications are unavoidable. As Powell et al. (2016) point out, it is "probably not a good idea to use any rain-type classification algorithm ... to 
confidently classify any particular single echo. Instead, one can apply the algorithm to large datasets and determine differences between the various categories of convection on average".

In this section we show two examples of the ECCO-V results from two very different field campaigns, the Southern Ocean Clouds Radiation Aerosol Transport Experimental Study (SOCRATES) and OTREC. The SOCRATES field campaign took place in January and February 2018 and consisted of 15 research flights over the Southern Ocean. It was designed to gather observations of the vertical distribution and properties of clouds and aerosols (McFarquhar et al. 2020). The clouds sampled during SOCRATES were mostly stratiform in nature. The example in Fig. 2, which was collected on January 29, 2018, from 01:09 to 01:53 UTC, shows a mid-to-high altitude stratiform cloud layer and a low-to-mid level precipitating cloud deck with embedded convective cells. The algorithm correctly identifies the mid convective cells embedded in the lower cloud layer. Towards the end of the time frame shown, the aircraft penetrated the top of the convective cells, and it was not possible to classify them into sub-categories. They keep the basic convective classification.
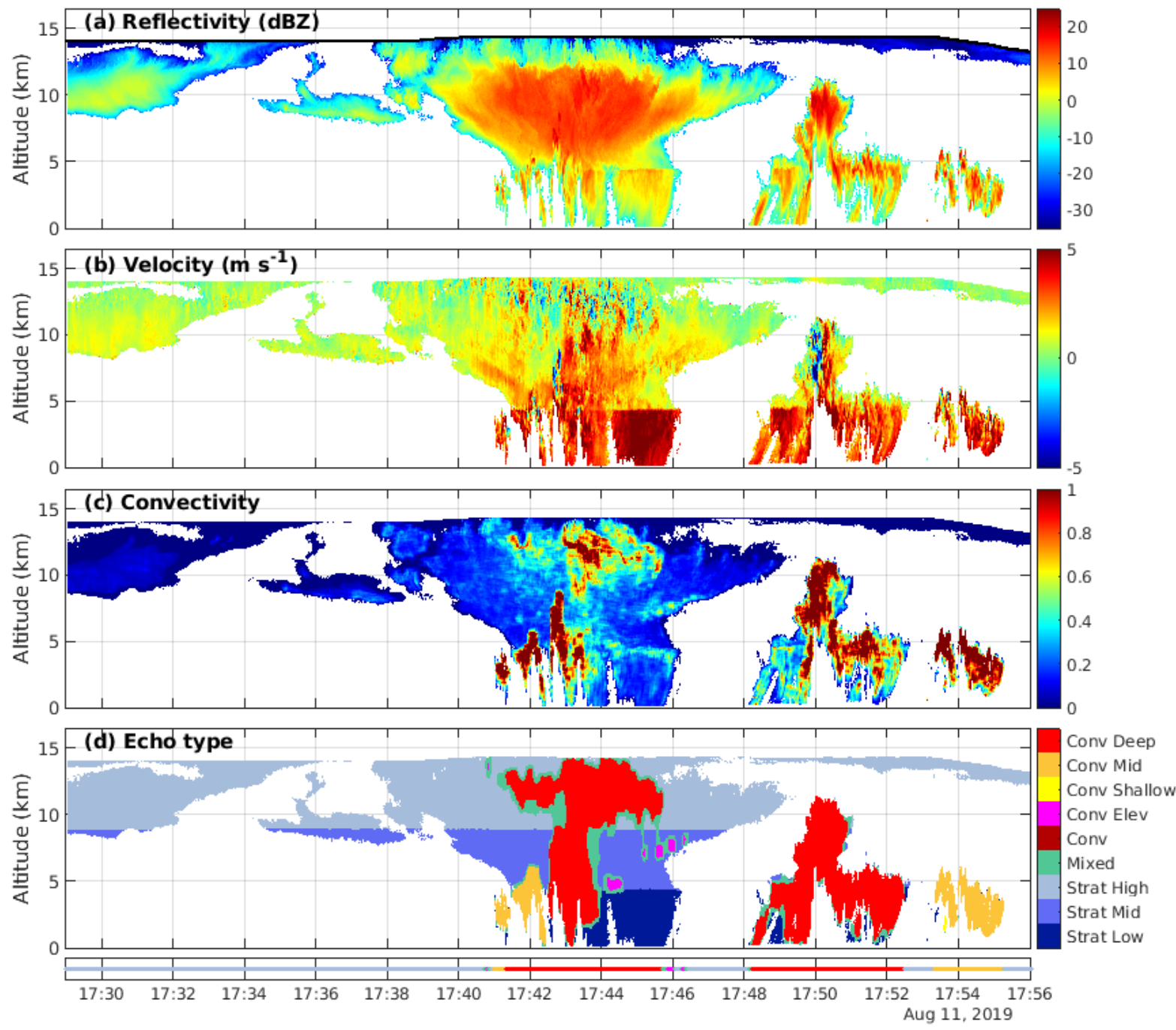

Fig. 3: As Fig. 2 but for data collected during OTREC on August 11, 2019, from 17:29 to 17:56 UTC. 
The OTREC field campaign, which took place from August to October, 2019, over the tropical East Pacific and the Caribbean Ocean, occurred in a very different environment. The sampled clouds and precipitation were associated with tropical convective systems of all scales and stages of development. The example shown in Fig. 3 shows a cross section through a mature thunderstorm (17:38 to 17:48 UTC) with a developing anvil cloud, newly developing thunderstorms in their early (17:54) and mid stages (17:50), and anvil clouds from nearby thunderstorms (17:29 to 17:38). ECCO-V identifies the anvil clouds as mid to high stratiform. It also captures the convective updraft region within the mature thunderstorm and labels it as a deep convective system. The part of the mature thunderstorm, which already developed a clear melting layer, is classified as stratiform (17:44 to 17:48). Small scale embedded convective echoes aloft are classified as elevated convective features. The classification of the newly developing cells as mid and deep reflect their stages of development.

This example demonstrates that the combination of TDBZ and TVEL is highly beneficial. Reflectivity provides the information on the strength and horizontal variability of the radar return signal while radial velocity identifies regions of significant vertical air motion, which leads to the identification of convective echo that may have been missed from $T D B Z$ alone (e.g. at high altitudes in the mature thunderstorm).

\subsection{CloudSat and MMCR}

To tune ECCO-V for space borne and ground based radars, input variables such as the length of the time segment, the scaling parameters, and the intensity of the morphological operations had to be adjusted. The melting layer and divergence level altitudes were provided as input to the algorithm and not derived from temperature, and the convectivity is based on reflectivity only as only $T D B Z$ is used. The input parameters were tuned specifically for the examples shown below and would likely require additional adjustments before ECCO-V could be deployed on a routine basis for these radars.

An example for CloudSat CPR observations is shown in Fig. 4. It shows an aging thunderstorm in its late, stratiform stage of development with a large anvil, and newly developing convective cells below that penetrate up into the anvil. This scene was captured on July 4, 2015, from 00:05:30 to 00:08:30 UTC over the northern Pacific Ocean $\left(-165^{\circ} \mathrm{W}\right.$, $\left.50^{\circ} \mathrm{N}\right)$. ECCO-V correctly identifies the stratiform clouds and precipitation, and classifies the convective cells according to their stages of development as mid and deep convective echoes.

The MMCR example observed a mostly stratiform cloud, which started precipitating as it passed over the radar, and a developing convective cell on August 17, 2008, between 13:45 and 14:35 UTC (Fig. 5). The developing cell (14:20 to 14:30) was classified as mid convective and the stratiform cloud as mid stratiform, while the stratiform precipitation was classified as low stratiform. The cloud above the precipitation showed elevated convective features. The robustness of the algorithm is demonstrated by this case. It performed well in conditions where a cloud is not captured at one specific stage, as is the case for air and space 
borne radars, but in conditions where cloud and precipitation change and develop while they are sampled by the ground based radar over a longer period of time.
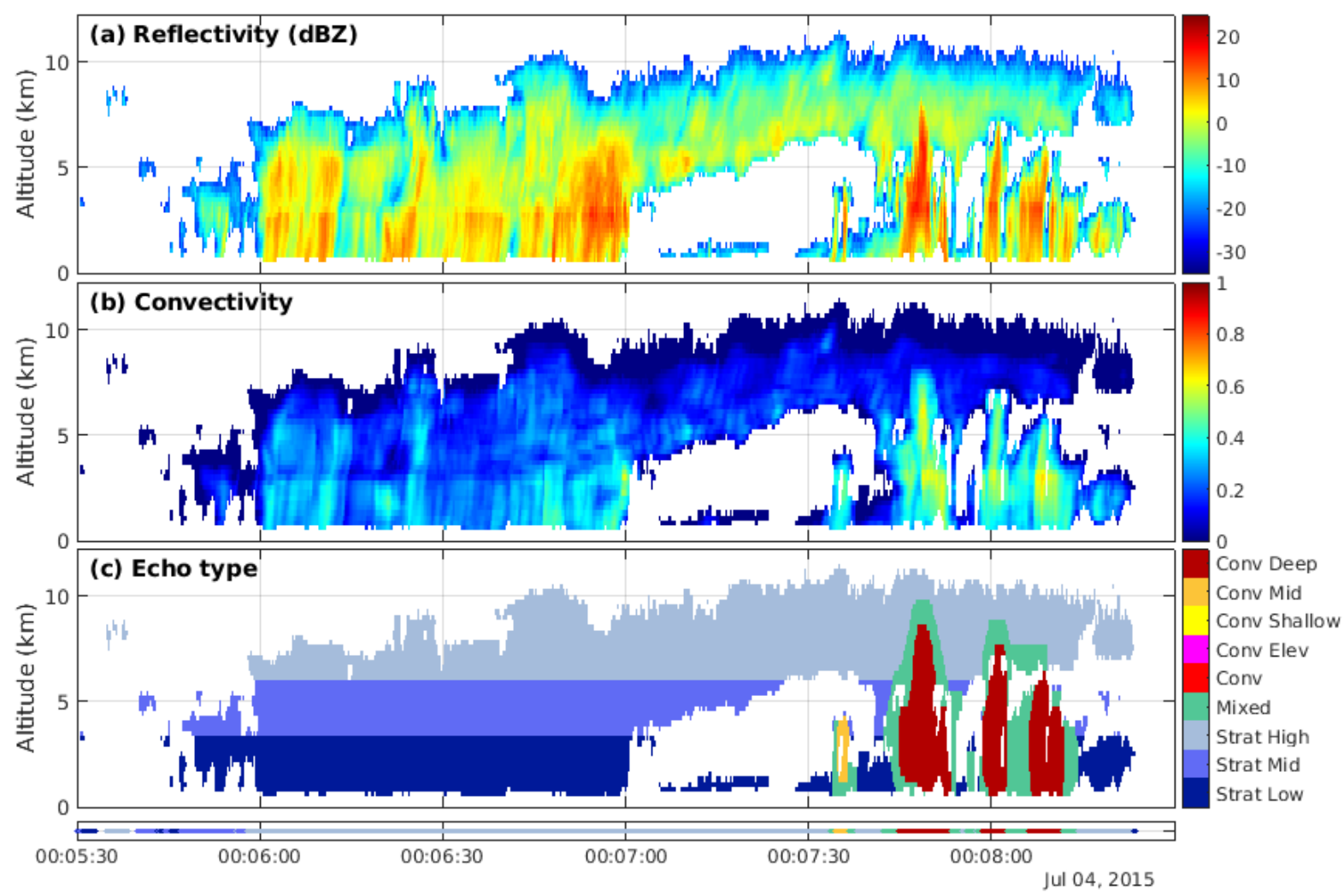

Fig. 4: (a) Reflectivity, (b) convectivity, and (c) echo type from CloudSat CPR data sampled on July 04, 2015 from 00:05:30 to 00:08:30 UTC over the northern Pacific Ocean.

\section{Conclusions}

The ECCO-3D method for convective/stratiform partitioning introduced by Dixon and Romatschke (2022) is adapted for vertically pointing radars (ECCO-V). Observations from four field campaigns collected by the airborne HIAPER Cloud Radar (HCR) are used to develop an algorithm that differentiates between convective and stratiform radar echoes not only on a vertical column by column basis, but also provides a vertically resolved classification field. ECCO-V is based on the calculation of reflectivity and velocity texture fields which provide information on both the horizontal homogeneity and heterogeneity of the observations. The texture fields are combined into a convectivity field, which provides a numeric measure of how convective or stratiform each sample point is. Convectivity can be used directly in suitable applications or it can be thresholded into the more traditional convective, stratiform, and in our case also mixed, radar echo categories. In a last step, the categories are refined to differentiate between low, mid, and high stratiform echoes, and shallow, mid, deep, and elevated convective echoes, and a mixed echo category, based on the melting layer and the divergence level altitudes, which are derived from temperature.

ECCO-V performs well and identifies not only isolated convective or stratiform clouds, but is particularly skilled in finding embedded convective features. For example, it 
differentiates between highly convective updrafts and aging stratiform regions within the same thunderstorm. It also finds newly developing convective cells underneath or even penetrating an elevated stratiform cloud layer. Elevated convective features, which are often embedded in stratiform clouds, are also identified. These capabilities highlight the importance of vertically resolved convective/stratiform echo type partitioning, in comparison to a simple one dimensional classification, which does not fully describe the complexity of multilayered cloud scenes.

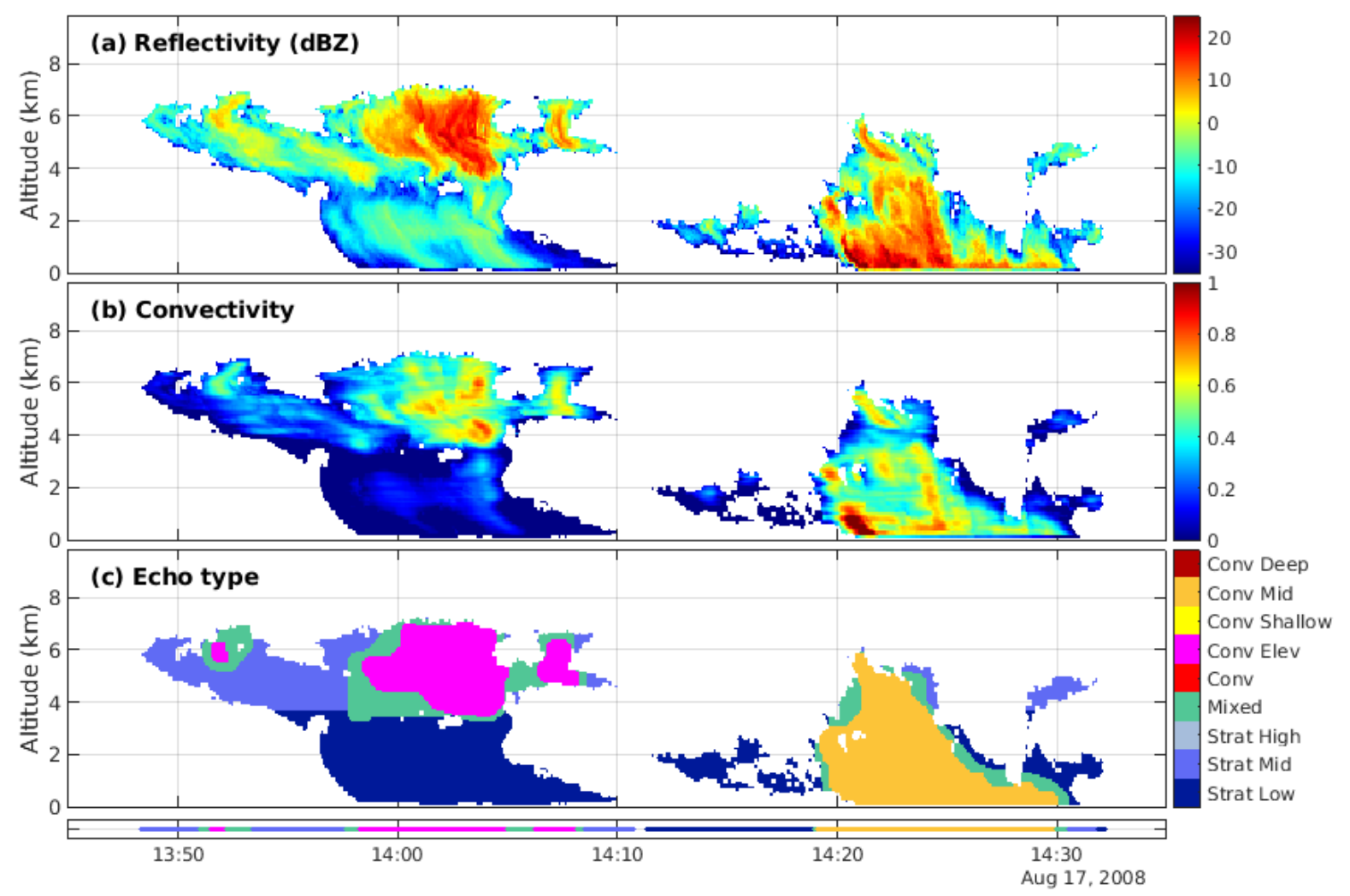

Fig. 5: As Figure CLOUDSAT but for data collected by MMCR on August 17, 2008, from 13:45 to $14: 35$ UTC.

ECCO-V is robust and was extensively tested in very different environments ranging from the tropics to the Southern Ocean. It is also highly adaptable and was easily adjusted for use in space borne and ground based vertically-pointing radar systems. In addition to its portability to different types of radars, it can also be adjusted for different uses and research requirements. The calculation of convectivity allows for highlighting of different characteristics of cloud and precipitation systems by adjusting the thresholding accordingly. ECCO-V is a versatile tool for cloud and precipitation research and we are confident that the convectivity and convective/stratiform echo type fields provided in the HCR datasets will lead to new insights into the mechanisms that govern cloud and precipitation formation and development. 


\section{Acknowledgments.}

This material is based upon work supported by the National Center for Atmospheric Research, which is a major facility sponsored by the National Science Foundation under Cooperative Agreement No. 1852977. We thank all members of the HCR team for the flawless operation of the radar during all HCR field campaigns. We thank John Hubbert and Brad Klotz for valuable comments on the manuscript.

\section{Data Availability Statement.}

HCR data from four major field campaigns is available in the EOL Field Data Archive https://data.eol.ucar.edu. The data for the CSET field campaign is available at https://doi.org/10.5065/D6CJ8BV7 (NCAR/EOL Remote Sensing Facility 2022a), the SOCRATES data at https://doi.org/10.5065/D68914PH (NCAR/EOL Remote Sensing Facility 2022b), the data for OTREC at https://doi.org/10.26023/V9DJ-7T9J-PE0S

(NCAR/EOL Remote Sensing Facility 2022c), and the SPICULE data at https://doi.org/10.26023/PGGK-MC4T-K70F (NCAR/EOL Remote Sensing Facility 2022d).

CloudSat data are available at https://www.cloudsat.cira.colostate.edu/. We use radar reflectivity from the 2B-GEOPROF product (Marchand et al. 2008).

MMCR data is available at https://doi.org/10.5439/1025228 (Atmospheric Radiation Measurement (ARM) user facility 2003).

\section{References}

Albrecht, B., and Coauthors, 2019: Cloud System Evolution in the Trades-CSET. Bull. Am. Meteorol. Soc., 100, 93-121, https://doi.org/10.1175/BAMS-D-17-0180.1.

Anagnostou, E. N., 2004: A convective/stratiform precipitation classification algorithm for volume scanning weather radar observations. Meteorol. Appl., 11, 291-300, https://doi.org/10.1017/S1350482704001409.

Atmospheric Radiation Measurement (ARM) user facility, 2003: Millimeter Wavelength Cloud Radar (MMCRMOM). ARM Data Center, Accessed 07 Dec 2021, https://doi.org/10.5439/1025228.

Awaka, J., T. Iguchi, H. Kumagai, and K. Okamoto, 1997: Rain type classification algorithm for TRMM Precipitation Radar. Geoscience and Remote Sensing, 1997. IGARSS '97. Remote Sensing - A Scientific Vision for Sustainable Development., 1997 IEEE International, Vol. 4 of, Geoscience and Remote Sensing, 1997. IGARSS '97. Remote Sensing - A Scientific Vision for Sustainable Development., 1997 IEEE International, 1633-1635. 
Biggerstaff, M. I., and S. A. Listemaa, 2000: An Improved Scheme for Convective/Stratiform Echo Classification Using Radar Reflectivity. J. Appl. Meteorol. Climatol., 39, 2129-2150, https://doi.org/10.1175/1520-0450(2001)040<2129:AISFCS>2.0.CO;2.

Dixon, M., and U. Romatschke, 2022: 3D Convective/Stratiform Echo Type Classification and Convectivity Retrieval from Radar Reflectivity. EarthArXiv, https://doi.org/10.31223/X58H02.

European Centre for Medium-Range Weather Forecasts, ERA5 Reanalysis (0.25 Degree Latitude-Longitude Grid). Research Data Archive at the National Center for Atmospheric Research, Computational and Information Systems Laboratory, Accessed 01 Jan 2021, https://doi.org/10.5065/BH6N-5N20.

Fuchs-Stone, Ž., D. J. Raymond, and S. Sentić, 2020: OTREC2019: Convection Over the East Pacific and Southwest Caribbean. Geophys. Res. Lett., 47, e2020GL087564, https://doi.org/10.1029/2020GL087564.

Haynes, J. M., 2018: CloudSat 2C-PRECIP-COLUMN Data Product Process Description and Interface Control Document.

http://www.cloudsat.cira.colostate.edu/sites/default/files/products/files/2C-PRECIP-COL UMN_PDICD.P1_R05.rev1_.pdf,.

Houze, R. A., 2014: Cloud Dynamics. Academic Press,.

Im, E., C. Wu, and S. L. Durden, 2005: Cloud profiling radar for the CloudSat mission. IEEE International Radar Conference, 2005., IEEE International Radar Conference, 2005., $483-486$.

Marchand, R., G. G. Mace, T. Ackerman, and G. Stephens, 2008: Hydrometeor Detection Using Cloudsat-An Earth-Orbiting 94-GHz Cloud Radar. J. Atmospheric Ocean. Technol., 25, 519-533, https://doi.org/10.1175/2007JTECHA1006.1.

McFarquhar, G. M., and Coauthors, 2020: Observations of clouds, aerosols, precipitation, and surface radiation over the Southern Ocean: An overview of CAPRICORN, MARCUS, MICRE and SOCRATES. Bull. Am. Meteorol. Soc., 1, 1-92, https://doi.org/10.1175/BAMS-D-20-0132.1.

NCAR/EOL Remote Sensing Facility, 2022: CSET: NCAR HCR radar moments data. Version 3.0. UCAR/NCAR - Earth Observing Laboratory. https://doi.org/10.5065/D6CJ8BV7.

NCAR/EOL Remote Sensing Facility, 2022: SOCRATES: NCAR HCR radar moments data. Version 3.0. UCAR/NCAR - Earth Observing Laboratory. https://doi.org/10.5065/D68914PH.

NCAR/EOL Remote Sensing Facility, 2022: OTREC: NCAR HCR radar moments data. Version 3.0. UCAR/NCAR - Earth Observing Laboratory. https://doi.org/10.26023/V9DJ-7T9J-PE0S. 
NCAR/EOL Remote Sensing Facility. 2022. SPICULE: NCAR HCR radar moments data. Version 1.1. UCAR/NCAR - Earth Observing Laboratory. https://doi.org/10.26023/PGGK-MC4T-K70F.

Powell, S. W., Robert A. Houze, and S. R. Brodzik, 2016: Rainfall-Type Categorization of Radar Echoes Using Polar Coordinate Reflectivity Data. J. Atmospheric Ocean. Technol., 33, 523-538, https://doi.org/10.1175/JTECH-D-15-0135.1.

Romatschke, U., 2021: Melting Layer Detection and Observation with the NCAR Airborne W-Band Radar. Remote Sens., 13, 1660, https://doi.org/10.3390/rs13091660.

—- M. Dixon, P. Tsai, E. Loew, J. Vivekanandan, J. Emmett, and R. Rilling, 2021: The NCAR airborne 94-GHz cloud radar: calibration and data processing. Data, 6, 66. https://doi.org/10.3390/data6060066.

Steiner, M., R. A. Houze, and S. E. Yuter, 1995: Climatological characterization of three-dimensional storm structure from operational radar and rain gauge data. J. Appl. Meteorol., 34, 1978-2007.

Vivekanandan, J., and Coauthors, 2015: A wing pod-based millimeter wavelength airborne cloud radar. Geosci. Instrum. Methods Data Syst., 4, 161-176, https://doi.org/10.5194/gi-4-161-2015.

Wang, Y., L. Tang, P.-L. Chang, and Y.-S. Tang, 2021: Separation of convective and stratiform precipitation using polarimetric radar data with a support vector machine method. Atmospheric Meas. Tech., 14, 185-197, https://doi.org/10.5194/amt-14-185-2021.

Yang, Y., X. Chen, and Y. Qi, 2013: Classification of convective/stratiform echoes in radar reflectivity observations using a fuzzy logic algorithm. J. Geophys. Res. Atmospheres, 118, 1896-1905, https://doi.org/10.1002/jgrd.50214. 